\title{
Editorial: Physical Review Accelerators and Beams: Open-Access Pioneer and Community Organizer
}

Since its start in 1998 Physical Review Accelerators and Beams (PRAB) has been an allelectronic journal, a daring novelty at the time and a testing ground for other Physical Review journals. Equally unheard of, thanks to external financial sponsorship, PRAB has been made available free of charge to both authors and readers around the world. As such, it is a pioneering "gold" open-access journal, far ahead of its time. Innovative and forward-looking, $P R A B$ rapidly established its reputation as the world's premier journal in accelerators and beams.

Accelerators have been a part of one-third of all physics Nobel Prizes awarded since 1939 [1] as well as being engines of discovery for chemistry, biology, and medicine. While members of the accelerator community make essential contributions to a broad range of sciences, "their peers are other accelerator scientists and their professional interests are related to accelerators and beams" [2]. Almost all accelerator experts are working at the nexus of universities, research centers, and industry, giving rise to unique collaboration models and research methodologies.

To better serve and nurture this community, in 1997 the APS Division of Physics of Beams (DPB) recommended establishing a scholarly, peer-reviewed journal devoted to the science and technology of accelerators and beams. It would cover the full breadth of accelerators and beams, publish quickly, circulate widely, and have an international editorial board and pool of referees [2].

Martin Blume, APS Editor in Chief at that time, understood the intimate connection between technology and the resulting science, and, departing from Physical Review tradition, he was willing to champion a journal covering all of accelerator research [2].

As a result, Physical Review Special Topics-Accelerators and Beams (PRST-AB) was approved by the APS Council in November 1997 and Robert H. Siemann of the Stanford Linear Accelerator Center, Stanford University, became the first editor. In 2016, the name was changed to Physical Review Accelerators and Beams to better integrate the journal into the Physical Review family.

The first PRST-AB article was published on 12 May 1998, and for more than a decade it was the fastest growing APS journal. At the same time, PRST-AB quickly became more international. At the start, $80 \%$ of the published articles originated in the Americas; this number decreased to $35 \%$ by 2017 . Currently the European and Asian regions contribute $45 \%$ and $21 \%$, respectively.

$P R A B$ continues to be completely free of charge for both authors and readers. It is an arrangement called "diamond" open access, made possible through the generous support of its sponsors, who recognize the importance of publishing accelerator science and technology. Initially eight large U.S. national laboratories supported the journal financially. Since then many other laboratories and research institutions - in the Americas, in Europe, and more recently in Asia, especially Japan, Korea, and China, as well as various accelerator conference seriesjoined as sponsors. Two years ago, $P R A B$ welcomed its first industrial sponsors-companies active in the fields of accelerator physics or accelerator technology. At present more than thirtyfive institutes and six companies sponsor $P R A B$. A list of all sponsors is available at journals.aps .org/prab/sponsors.

With this support, day-to-day operations are coordinated by a Lead Editor, three Associate Editors, and a Journal Manager. The Editors are assisted by an Editorial Board-a valuable resource for discussions of policies and new initiatives. Board members also serve as referees in cases where there are contentions or questions on which the Editors need advice. These board members are well-respected accelerator scientists, who represent different research specialties, strike a balance between universities and large laboratories, and connect $P R A B$ internationally. A list of present $P R A B$ staff and Editorial Board members is posted at journals.aps.org/prab/ staff\#ed. 
In a further innovation, the APS DPB and the European Physical Society Accelerators Group share responsibility for the health and vitality of the journal by advising on the membership of the Editorial Board, and by encouraging scholarly publication in accelerator science and technology.

In response to increasing interest and demand as well as to better cover topics at the boundaries between disciplines, $P R A B$ has recently introduced new dedicated topic sections (go.aps.org/2Gbs4dz). And like other APS journals, $P R A B$ highlights important articles in the form of "Editors' Suggestions." A selected few have been covered by the APS commentary journal Physics. In addition, aesthetically attractive pictures, one per month, appear on the journal webpage with links to the corresponding articles.

Through special editions, invited contributions, articles related to the APS Robert R. Wilson Prize for Achievement in Accelerator Physics, editorial outreach, tutorials, and Editorial Board meetings during the International Particle Accelerator Conferences, PRAB has become an important "Community Organizer," thereby realizing one of the intentions of its founders. For the coming years, $P R A B$ looks forward to further transforming scientific publication in the field of accelerators.

We welcome your feedback and suggestions (prab@aps.org).

[1] E. F. Haussecker and A. W. Chao, Influence of accelerator science on physics research, ICFA Beam Dyn. Newsl. 53, 11 (2010).

[2] R. Siemann, Essay: Accelerators, beams and physical review special topics-accelerators and beams, Phys. Rev. ST Accel. Beams 11, 050003 (2008).

Frank Zimmermann

Published 3 April 2018

DOI: 10.1103/PhysRevAccelBeams.21.040001

PRAB Lead Editor Frank Zimmermann is a Senior Scientist at the European Organization for Nuclear Research (CERN) in Geneva, Switzerland, a Fellow of the APS, and a Board Member of the Accelerator Group in the European Physical Society.

[This information also appears in the April issue of APS News.] 